\title{
SEISMIC FRAGILITY CHARACTERISTICS OF STRUCTURAL POPULATIONS WITH IRREGULARITIES
}

\author{
JUNWON SEO \\ Department of Civil and Environmental Engineering, South Dakota State University, USA.
}

\begin{abstract}
Earthquakes result in substantial structural damage of a number of structures across a region. Many studies have made an effort to examine regional seismic damage and vulnerability to different structure types, e.g. buildings and bridges, using varying computational methodologies. This paper focuses on the use of Response Surface Metamodels (RSMs) in conjunction with Monte Carlo Simulations (MCSs) to quantify probabilistic seismic performance for different classes of structural populations with irregularities, including irregular steel buildings and steel girder bridges. As part of the regional vulnerability study, each of the selected classes is constructed based upon the appropriate experimental design technique, i.e. the Central Composite Design (CCD), and the responses of each class subjected to multiple ground motions are captured during the nonlinear time history analyses of an individual computational model. Then, a RSM for each class is established by performing a least-square regression analysis within the considered CCD space. Seismic fragility curves are generated by means of the joint RSM-MCS enabling to treat uncertainties regarding overall configuration irregularities and additional structural parameters considered significant for each class. The influence of the irregularity parameters on seismic vulnerability for each class is investigated by comparison of the resulting fragilities. Results reveal that the RSM-MCS is able to efficiently assess seismic vulnerability of each class and directly examine the parameters' influence on corresponding behaviours.

Keywords: irregularities, metamodel, seismic response, structural populations, vulnerability.
\end{abstract}

\section{INTRODUCTION}

A number of structures across susceptible regions to earthquakes have been substantially damaged, resulting in economic and human losses. To make wise decisions for enhanced earthquake mitigations, it is required that seismic losses of structures with regional characteristics data (e.g. structural dimensions) be appropriately predicted. It is well known that the most efficient methodology to estimate seismic losses of structures accounting for uncertainty in seismic loadings and structural capacities is the seismic fragility analysis [1-4]. Numerous studies have been performed to investigate regional seismic damage and fragility to various structure types, e.g. buildings and bridges, via different computational or statistical modelling techniques [5-8]. Different metamodels created with seismic responses computed by performing nonlinear time history analyses of structural models have been utilized to estimate seismic fragilities of structures with an aid of reliability techniques $[4,9]$. Recently, metamodels have been used to estimate seismic restoration costs of structural populations [10].

This paper is intended to evaluate the feasibility of Response Surface Metamodels (RSMs) classified as a metamodel type for probabilistic seismic performance quantification for structural populations with irregularities. RSMs coupled with Monte Carlo Simulations (MCSs) were applied to two different classes for irregular structures: 1) steel moment resisting frame buildings and curved steel girder bridges. As part of the study, sample combinations for each structure class were constructed based upon the Central Composite Design (CCD), and the responses of each combination subjected to ground motions were obtained during the nonlinear time history analyses. Then, a RSM for each class is established by performing a least-square regression analysis within the considered CCD combination space. Seismic fragility curves for each class were generated by means of the joint RSM-MCS enabling to treat uncertainties 
regarding structural irregularities and other parameters considered significant on seismic behaviours. The influence of the considered irregular parameters on seismic vulnerability per class was investigated by comparison of the resulting fragility curves.

\section{APPROACH}

A seismic RSM is typically defined as a polynomial regression function that can be created by fitting a dataset consisting of structural characteristics and seismic responses. The RSMs can be used not only to forecast seismic responses of structure(s), but also to generate their fragility curves at low-computational costs in conjunction with reliability techniques. Details on the RSM-based seismic response prediction and fragility curve generation are provided in the following subsections.

\subsection{Seismic response prediction}

The use of a second-order polynomial RSM function is a traditional approach to predict seismic response quantities. The RSM can be expressed as indicated below [11].

$$
y=\beta_{0}+\sum_{i=1}^{k} \beta_{i} x_{i}+\sum_{i=1}^{k} \beta_{i i} x_{i}^{2}+\sum_{i=1}^{k-1} \sum_{j>i}^{k} \beta_{i j} x_{i} x_{j}+\varepsilon
$$

where $y$ is the estimated seismic response; $x_{i}, x_{j}$ are input variables, such as irregularity parameters; $\beta_{0}, \beta_{i}, \beta_{i j}, \beta_{i i}$ are coefficients to be determined from experimental sets of seismic response quantities and input variables; and $k$ is the number of input variables. It should be noted that the minimum number of experimental sets required for determining RSM coefficients is normally equivalent to the number of the input variables [11].

To predict seismic response quantities of structures, RSMs consisting of input and output variables should be created at first. The input variables are composed of structural, material, and geometrical parameters necessary for the construction of analytical models associated with their variation in structures located across a region. A range of values for each input variable can be determined by examining design plans for structures or reviewing findings from the past studies. Experimental designs, including CCD, can be employed to establish possible combinations, which are structural models.

Based on each input combination with the corresponding values, a structural model can be developed with multiple frame elements using Finite Element Modelling (FEM) software. To account for uncertainty in potential earthquake scenarios associated with seismic resistance which varies depending on structural characteristics, multiple ground motions need to be considered. One ground motion at a time can be applied to each of the generated FEM model, and then the corresponding seismic response quantities can be determined by performing its seismic analysis. Note that the quantities can be categorized as output variables. The outputs can be incorporated into the inputs to complete a matrix. The matrix is used as the basis to be run by a least square regression analysis for the development of RSMs enabling the seismic response prediction.

\subsection{Seismic fragility curve generation}

Prior to providing a procedure for the generation of seismic fragility curves through RSMs, it is worth noting its physical definition. The seismic fragility curve for structures is a conditional probability, providing the probability of failure or exceedance that a structure will 
exceed a particular state of damage given ground motion intensity levels [1-4]. This fragility curve is mathematically expressed in Equation (2):

$$
P_{f}=\Phi\left(\frac{\ln \left(\frac{S_{D}}{S_{C}}\right)}{\sqrt{\beta_{d}^{2}+\beta_{c}^{2}}}\right)
$$

where $P_{f}$ is the exceedance probability, $S_{c}$ is the median value of the seismic resistance at a given damage state, $\beta \mathrm{c}$ is the dispersion or lognormal standard deviation of the seismic resistance, $S_{d}$ is the computed seismic demand at a chosen ground motion intensity parameter, $\beta_{d}$ is the logarithmic standard deviation for the demand and $\Phi$ is the standard normal distribution function [5].

Based on the notion for the generation of the standard fragility curves, seismic fragility curves can be created using RSMs in conjunction with MCSs. MCSs with randomness within a probabilistic characteristic for each input variable can be conducted to compute all possible seismic responses using RSMs. The probabilistic characteristics can be quantified in terms of probability density functions. The likelihood of the response exceeding a particular damage state is determined from numerous seismic response outputs obtained from the MCS with RSMs. This procedure provides a single probability value which is part of a fragility curve at a given ground motion intensity level; thus, this can be iterated for the other earthquake intensity levels to generate a complete fragility curve. More information on the RSM-based fragility curve generation can be discovered in the past studies [1, 4].

\section{APPLICATION TO IRREGULAR STRUCTURE POPULATION}

The RSM-MCS approach was applied to irregular structure populations: 1) steel moment-resisting building structures and 2) curved steel girder bridge structures. To assess the seismic vulnerability of each of the structure populations, fragility curves considering geometrical and structural characteristics for each class were created using the RSM-MCS. Then, the effect of the input variables focusing on irregularity parameters on the vulnerability for each class was examined by performing a comparative study of respective fragility curves. The generation of seismic fragility curves and the completion of cursory sensitivity analyses for each class are detailed as follows.

\subsection{Steel building structures}

\subsubsection{Building characteristics}

As shown in Fig. 1a, steel moment-resisting building structures in Shelby County, TN, in the United States, which is located in the New Madrid Seismic Zone (NMSZ) were chosen for this application. Note that the NMSZ is considered the most seismically active area in the Central United States as a series of past high-intensity earthquakes and aftershocks occurred. As a result of infrequent seismic events, the majority of existing structures have been only designed considering the combined gravity and wind loadings without seismic details. Specifically, L-shaped moment-resisting frame structures considered irregular buildings in plan were selected to analyse their seismic vulnerability in this region. As illustrated in Fig. 1b, the most feasible L-shaped plan configurations that were determined from the past work [1] were 


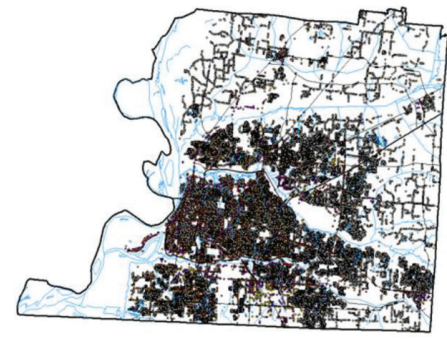

(a)

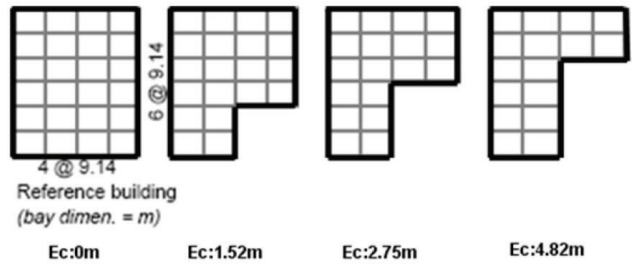

(b)

Figure 1: Region of interest. (a) Building stocks in Shelby County, TN; (b) most feasible L-shaped configurations [1].

utilized in this study. Based on the examination of building inventory in the region of interest (see Table 1), most buildings are made up for industrial occupancy use and consist of less than five stories and different built years. Hence, the story of structures was limited to five-story office buildings with a variety of L-shapes and built years.

After the building shapes in plan were determined, potential variables for the selected structures, which have a significant impact on regional seismic response and fragility, were considered to include their uncertainty. These variables included earthquake direction, steel yield strength, damping ratio, bottom floor height, year built, and eccentricity. Forty-three experimental design combinations (i.e. 43 buildings), which served as the basis for the development of FEM building models, were constructed based upon the CCD with the seven variables. When building the CCD-based combinations, upper, central, and low values corresponding to each variable are required. Detailed information (i.e. values) for each variable was obtained from the past study [1].

\subsubsection{Structural design and seismic simulation}

Each building out of 43 combinations was designed according to appropriate building design codes. For example, a building with pre-1970 built year that was compatible to the non-seismic design was designed following the 1969 Southern Standard Building Code (SSBC) [13] with consideration of only gravity and wind loads. Another example is that a building with post-1990 year built was designed according to 1991 Southern Building Code Congress International (SBCCI) [14]. For the non-seismic building design, an example of exterior and interior columns and beams selected according to the SSBC building design requirements was shown in Table 2.

Table 1: Building inventory data in Shelby County [12].

\begin{tabular}{llll}
\hline Occupancy & Industrial & Residential & Others \\
& $97.3 \%$ & $2.1 \%$ & $0.6 \%$ \\
Year Built & Pre-1970 & $1970-1990$ & Post-1990 \\
& $58.1 \%$ & $32 \%$ & $9.9 \%$ \\
Number of stories & Less than 5 & $6-10$ & More than 11 \\
& $99.9 \%$ & $0 \%$ & $0.1 \%$ \\
\hline
\end{tabular}


Table 2: Structural frames for the example LSMF building designed with SSBC 1969.

\begin{tabular}{llll}
\hline \multirow{5}{*}{ Story } & \multicolumn{2}{l}{ Moment resisting frames } \\
\cline { 2 - 4 } & E. Columns & I. Columns & Beams \\
\hline 1 & W14X111 & W14X111 & W24X76 \\
2 & W14X111 & W14X111 & W24X76 \\
3 & W14X95 & W14X95 & W24X76 \\
4 & W14X95 & W14X95 & W24X76 \\
5 & W14X68 & W14X68 & W24X68 \\
\hline
\end{tabular}

An analytical 3D model to each building was generated using frame elements available in Zeus-NL [15] accounting for material and geometric nonlinearities. Geometric and material properties for columns and beams were described using the sections selected from the structural design process. It was assumed that the connections between beam and columns that were idealized as simple joints were fully fixed at all rotational and translational directions. Lumped masses were assigned to each element enabling seismic analyses. Representative analytical models for both rectangular- and L-shaped buildings can be seen in Fig. 2. The rectangular building was used as a benchmarking regular building, so as to compare its fragilities against those resulting from buildings with different L-shapes.

To evaluate seismic vulnerability of the structures subjected to various potential earthquake scenarios, synthetic ground motions that were generated by Rix and Fernandez-Leon [16] for the region were used as input loads in the models. The ground motion pool was created based upon the soil and other site features in the region where there has been a lack of actual ground motion data. In detail, 30 ground motions having different magnitudes and hypocentral distances that were extracted out of the pool were applied to each model. Spectral acceleration $\left(S_{a}\right)$ curves representing the input ground motions can be seen in Fig. 3, and

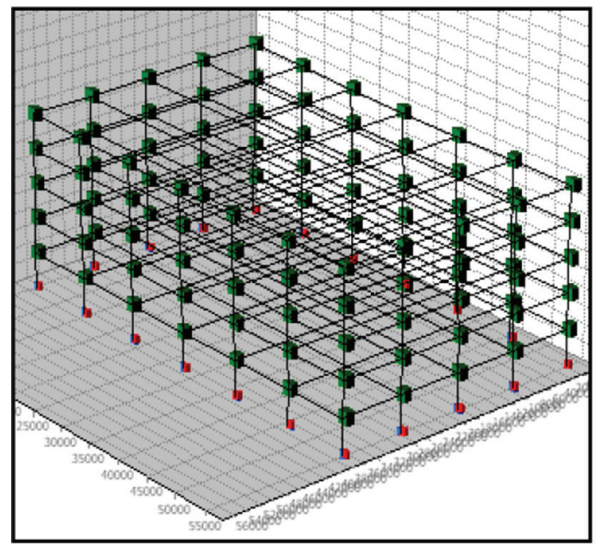

(a)

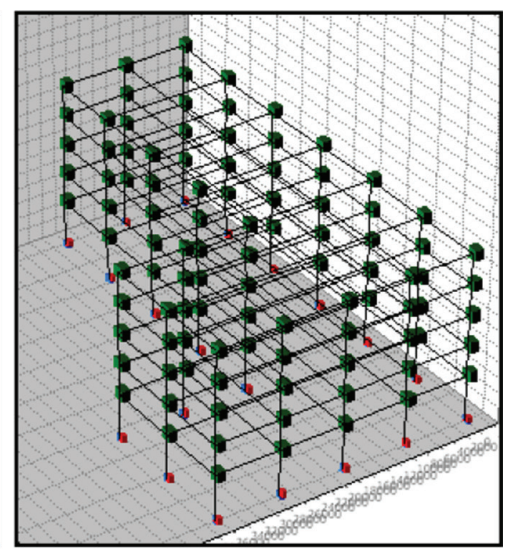

(b)

Figure 2: Sample 3D models. (a) Rectangular-shaped building model; (b) L-shaped building model. 


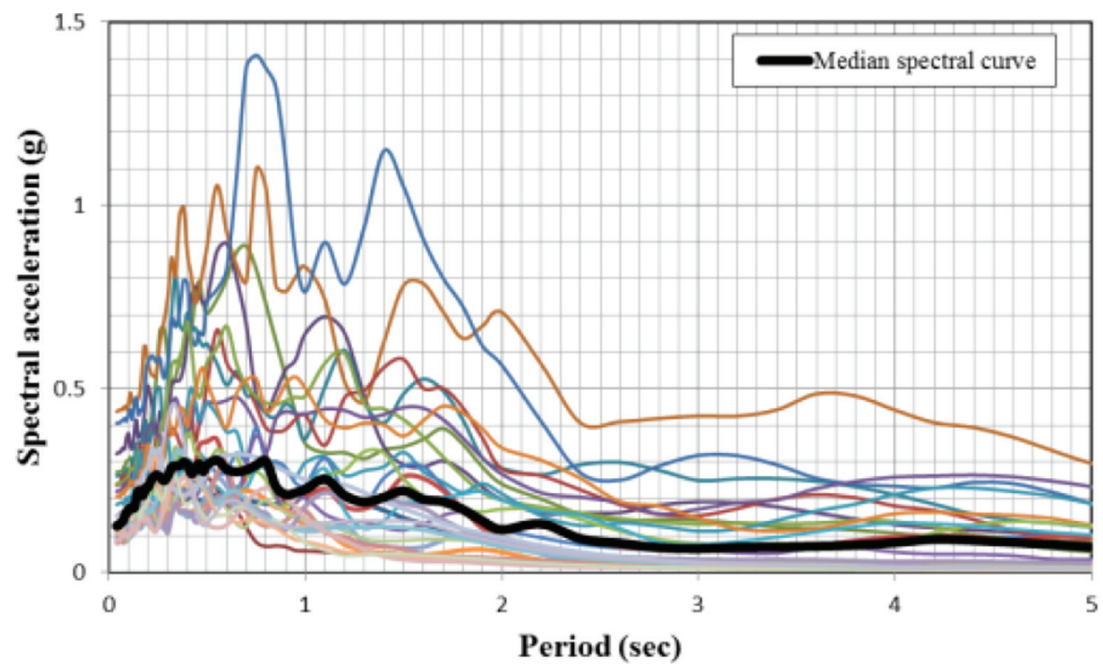

Figure 3: Spectral acceleration $[1,16]$.

the corresponding median value is also plotted in this figure. It appears that the $S_{a}$ values vary depending on ground motion characteristics.

Nonlinear time history analysis of each model loaded with one from the ground motion pool was separately performed to estimate its seismic response. Translational displacements resulting from the ground motions were captured during the seismic analyses, and maximum inter-story drift ratios that have been commonly used as a seismic behaviour assessment indicator were calculated using the following equation:

$$
\text { Maximum Inter }- \text { Story Drift Ratio }=\max _{\forall(x, z, t), i} \mid\left(\frac{\delta_{(x, t), i}}{h_{i}}, \frac{\delta_{(z, t), i}}{h_{i}}\right)
$$

where $\delta_{(x, t), i}$ and $\delta_{(z, t), i}$ are the translational displacement responses in the strong and weak directions at time $t$ at the $i^{t h}$ floor when performing the nonlinear time history analysis of the model; $h_{i}$ is inter-story height of the $i^{\text {th }}$ floor [1]. By running the seismic analyses, outputs, which are the max inter-story drift ratios, corresponding to each of the 43 input combinations were obtained.

\subsubsection{Building fragilities}

A regression analysis for a complete matrix consisting of all inputs and outputs was carried out to construct a RSM to estimate the max inter story drift ratios and generate fragility curves for the structures. The RSM function was able to approximate the drifts for the various structures resulting from a certain level of $S_{a}$. MCSs with probability density functions of each input variable that were assumed in the past work [1] were performed on the RSM function. Probability, which exceeds a drift ratio limit at a certain damage state were determined from the responses from the joint MCS-RSM simulations, resulting in a single fragility point given a $S_{a}$. In this application, three damage limit states, including IO=immediate occupancy, $\mathrm{LS}=$ life safety, $\mathrm{CP}=$ collapse prevention, that have been widespread in the fragility assessment of moment-resisting structures were used. By performing the described process multiple times, a number of probability values at different levels of $S_{a}$ were obtained, and these points 
were plotted to complete a fragility curve for each damage state. The overall fragility curves of the structures are illustrated in Fig. 4a. Detailed information for the generation of fragility curves can be found elsewhere [1].

To assess the influence of planar building irregularity that can be expressed in terms of eccentricity on seismic fragilities of the structure population, specific fragility curves (see Fig. 4 b) accounting for variability in eccentricity (i.e. $0 \mathrm{~m}, 2.8 \mathrm{~m}$, and $4.82 \mathrm{~m}$ ) were produced using the MCS-RSMs. A particular eccentricity value used as one governing variable was

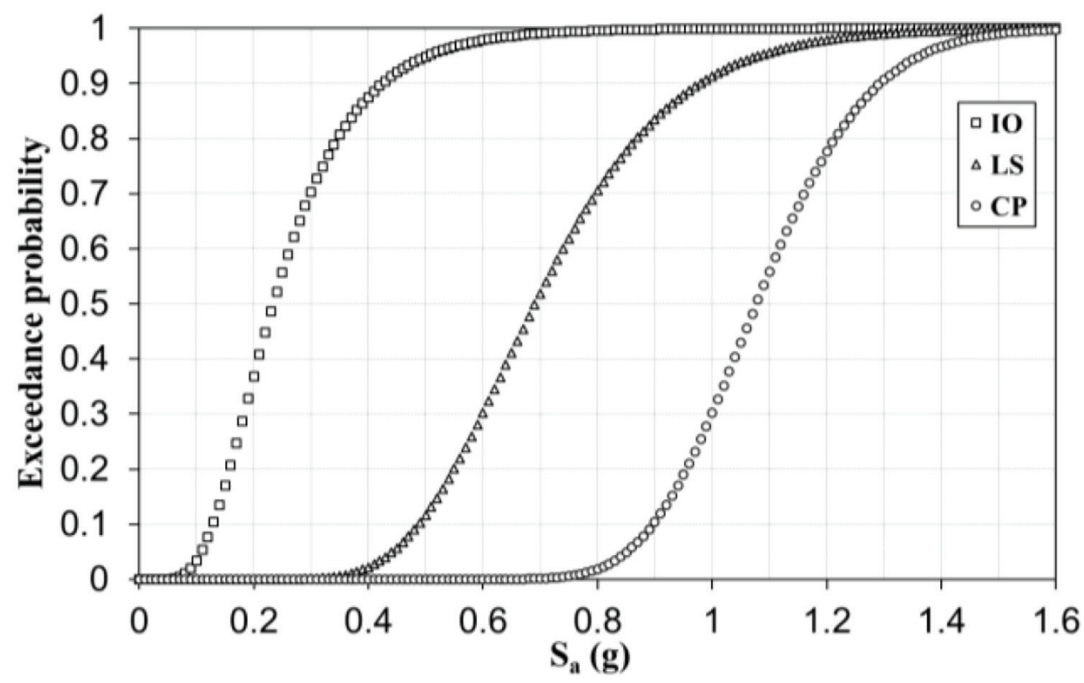

(a)

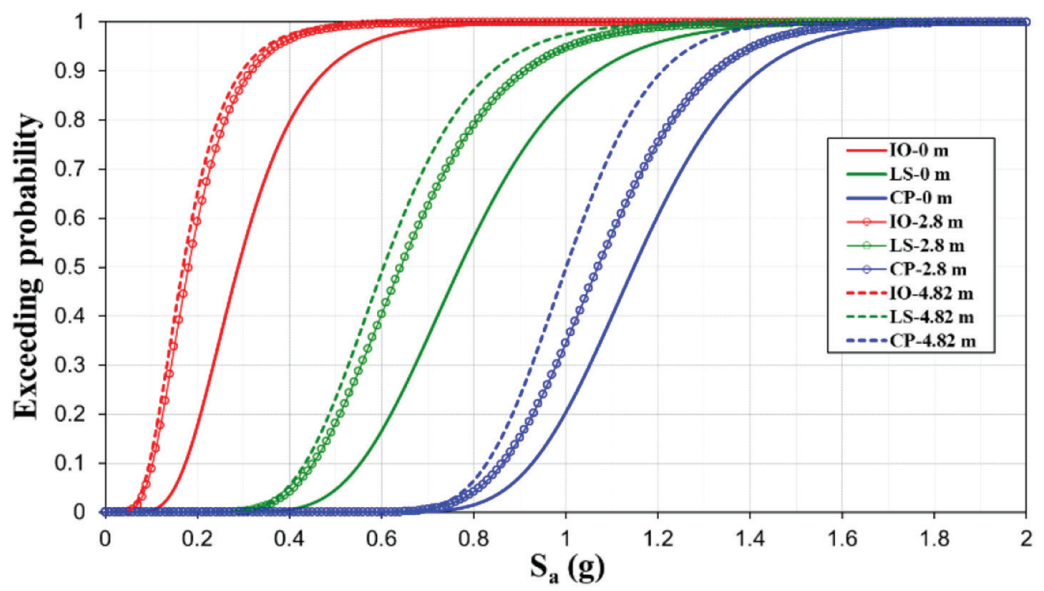

(b)

Figure 4: Sample building fragilities. (a) Overall curves; (b) specific curves with irregularities [1]. 
treated as the fixed one during the simulations, but the remainder of variables were considered randomly changing variables within the assumed probability range. The resulting curves for three different values of eccentricity were visually compared to explore the effect of building irregularity on seismic vulnerability at each damage state over a range of $S_{a}$. It appears that vulnerability for the structures over three damage states becomes higher due to an increase in seismic torisonal responses captured from the seismic analyses of buildings with the increase in planar eccentricity.

\subsection{Steel bridge structures}

\subsubsection{Bridge characteristics}

The proposed approach was also applied to horizontally, curved steel I-girder bridges which are classified as irregular bridges, in an attempt to look into their fragilities. For the bridge application, 99 curved steel bridges located across the States of Pennsylvania, Maryland, and New York in the United States were collected and examined. For the inventory analysis of the bridges, the bridge design plans were directly obtained from the respective Departments of Transportation (DOTs). Statistics for significantly structural bridge variables, including number of spans, maximum span length, deck width, maximum column height, radius of curvature, girder spacing, and cross-frame spacing, are listed in Table 3. This table indicates that the majority of the bridges have two spans. The variables with the statistical information were used as input variables for the CCD-based RSM establishment as created in the building fragility study. To account for uncertainties in material and other features for the bridges, steel and concrete strengths and Young's moduli and damping ratio were included in the RSM establishment as well.

\subsubsection{Structural modelling and seismic analysis}

3D analytical models of the bridges made with different values for each input variable were generated using OpenSees program [17], reflecting their material and geometric nonlinearity. The models were based upon the recommended modelling techniques from the past work [18]. The girders and concrete deck in a superstructure system were idealized using BeamColumn elements available in OpenSees, while pier columns and caps, abutments, and footings were modelled with the OpenSees displacement-based BeamColumn elements. The representative ground motions that were arbitrarily selected, considering a broad range of

Table 3: Statistics for significant bridge variables of the considered curved bridges [4].

\begin{tabular}{lllll}
\hline Bridge variables & Mean & $\begin{array}{l}\text { Standard } \\
\text { deviation }\end{array}$ & Median & Mode \\
\hline Number of spans & 2.53 & 1.73 & 1.5 & 2 \\
Maximum span length, m & 43.8 & 13.7 & 43.1 & Not Unique \\
Deck width, m & 12.9 & 4.4 & 12.2 & 12.8 \\
Maximum column height, m & 6.5 & 4.3 & 5.3 & 4.6 \\
Radius of curvature, m & 513.5 & 542.9 & 304.6 & 250 \\
Girder spacing, m & 2.6 & 0.4 & 2.5 & Not Unique \\
Cross-frame spacing, m & 5.4 & 1.2 & 5.2 & 7.3 \\
\hline
\end{tabular}


peak ground accelerations (PGAs), from the ground motion pool used in the building fragility analysis was used for the bridge analysis. Nonlinear time history analyses for each model subjected to each ground motions were completed, and seismic responses, including maximum column curvature ductility and bearing and abutment deformations, were extracted during each analysis. These peak responses were considered outputs to each model. The RSM formula was developed within the combined input and output matrix by performing its least-square regression analysis. More details on the modelling approach and the RSM development are able to be found in the past studies [3-4].

\subsubsection{Bridge fragilities}

This section involves creation of seismic fragility curves for the bridge population using the RSM formula in conjunction with MCSs. The probability of the estimated seismic response using the RSM to surpass a quantitative limit was calculated at each of the damage limit states, including slight, moderate, extensive, and complete that were described and determined by Federal Emergency Management Agency (FEMA). Specifically, the probability was determined using the RSM-MCS results under the probability density distribution for each input variable. This point was deemed to be a probability or fragility value at a given, particular PGA. This procedure was repeated over an entire range of PGAs, and then a whole fragility curve was built.

To examine geometrical irregularity effects of the curved bridge population on seismic vulnerability, a comparison between fragility curves of two bridges per damage state, including the almost straight bridge with radius of curvature $3036 \mathrm{~m}$ and tight curved bridge with $871 \mathrm{~m}$ curvature, was made as shown in Fig. 5. It shows that the fragilities for the tight curved bridge which has more geometrical irregularity than the almost straight bridge are higher than those for the straight one. It can be interpreted that the curved bridge is more vulnerable to seismic events than straight bridge because the increase in radius of curvature resulted in an increase in global seismic torsional responses, leading to higher seismic fragility.

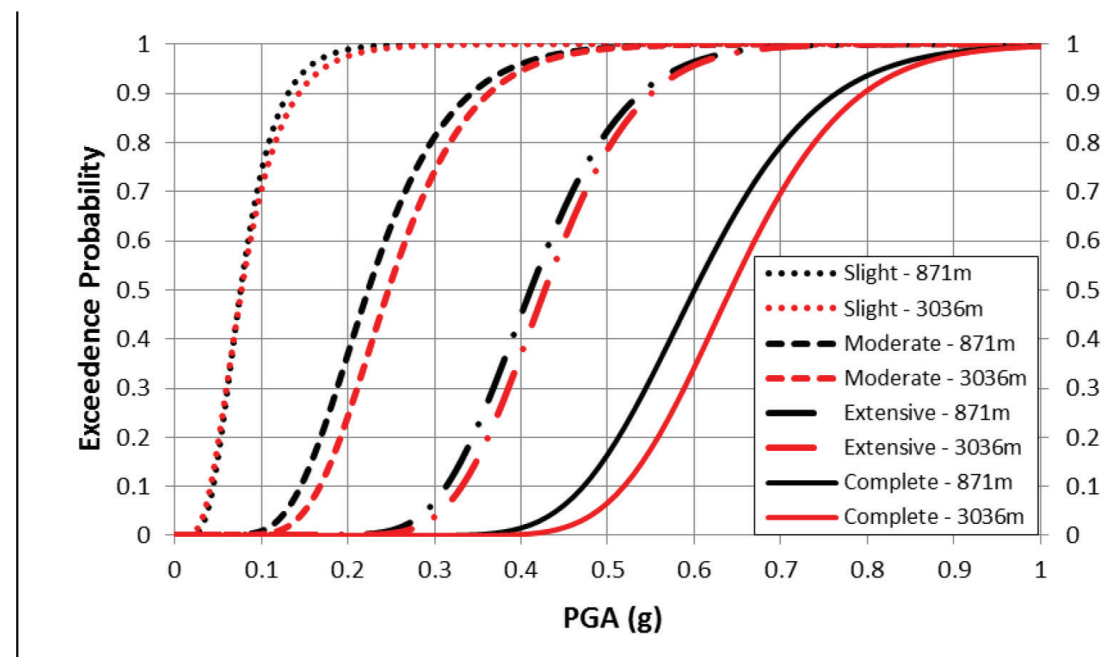

Figure 5: Comparison between fragility curves of two bridges having a radius of curvature of $871 \mathrm{~m}$ and $3036 \mathrm{~m} \mathrm{[4].}$ 


\section{CONCLUSIONS}

Integrated RSMs and MCS methodology was implemented to demonstrate that seismic fragility curves for structural populations with irregularities may be generated in an efficient manner. The methodology was applied to two classes of irregular structures, including steel momentresisting framing building structures having planar irregularities and horizontally curved steel I-girder bridges. It has been proven that the seismic vulnerabilities of each class were rapidly assessed in the form of fragility curves that were built by performing efficient numbers of simulations through the RSM-MCS process. This methodology helped not only to enhance the computational efficiency to examine both building and bridge vulnerabilities to earthquakes, but also to explore the effects of structural irregularities on each. For further implementation of this methodology, this should be validated with either experimental or empirical fragility data that can be probabilistically produced by performing structural damage data resulting from actual ground motions.

\section{REFERENCES}

[1] Seo, J., Dueñas-Osorio, L., Craig, J.I. \& Goodno, B.J., Metamodel-based regional vulnerability estimate of irregular steel moment-frame structures subjected to earthquake events. Engineering Structures, 45, pp. 585-597, 2012. https://doi.org/10.1016/j.engstruct.2012.07.003

[2] Seo, J. \& Linzell, D.G., Horizontally curved steel bridge seismic vulnerability assessment. Engineering Structures, 34, pp. 21-32, 2012. https://doi.org/10.1016/j.engstruct.2011.09.008

[3] Seo, J. \& Linzell, D.G., Nonlinear seismic response and parametric examination of horizontally curved steel bridges using 3D computational models. ASCE Journal of Bridge Engineering, 18(3), pp. 220-231, 2013. https://doi.org/10.1061/(asce)be.1943-5592.0000345

[4] Seo, J. \& Linzell, D.G., Use of response surface metamodels to generate system level fragilities for existing curved steel bridges. Engineering Structures, 52, pp. 642-653, 2013. https://doi.org/10.1016/j.engstruct.2013.03.023

[5] Rogers, L.P., \& Seo, J., Vulnerability sensitivity of curved precast-concrete I-girder bridges with various configurations subjected to multiple ground motions. ASCE Journal of Bridge Engineering, 22(2), pp. 04016118-1-19, 2017. https://doi.org/10.1061/(asce)be.1943-5592.0000973

[6] Park, J., Towashiraporn, P., Craig, J.I. \& Goodno, B.J., Seismic fragility analysis of low-rise unreinforced masonry structures. Engineering Structures, 31(1), pp. 125-137, 2009. https://doi.org/10.1016/j.engstruct.2008.07.021

[7] Jeon, J.S., DesRoches, R., Kim, T. \& Choi, E., Geometric parameters affecting seismic fragilities of curved multi-frame concrete box-girder bridges with integral abutments. Engineering Structures, 122, pp. 121-143, 2016. https://doi.org/10.1016/j.engstruct.2016.04.037

[8] Jeon, J.S., DesRoches, R. \& Lee, D.H. Post-repair effect of column jackets on aftershock fragilities of damaged RC bridges subjected to successive earthquakes. Earthquake Engineering and Structural Dynamics, 45(7), pp. 1149-1168, 2016. https://doi.org/10.1002/eqe.2700 
[9] Ghosh, J., Padgett, J.E. \& Dueñas-Osorio, L., Surrogate modeling and failure surface visualization for efficient seismic vulnerability assessment of highway bridges. Probabilistic Engineering Mechanics, 34, pp. 189-199, 2013. https://doi.org/10.1016/j.probengmech.2013.09.003

[10] Seo, J. \& Park, H., Probabilistic seismic restoration cost estimation for transportation infrastructure portfolios with an emphasis on curved steel I-girder bridges. Structural Safety, 65, pp. 27-34, 2017. https://doi.org/10.1016/j.strusafe.2016.12.002

[11] Wu, C.F.J. \& Hamada, M., Experiments-Planning, Analysis and Parameter Design Optimization, John Wiley \& Sons, pp. 760, 2000.

[12] Muthukumar, S., The application of advanced inventory techniques in urban inventory data development of earthquake risk modeling and mitigation in Mid-America. $\mathrm{PhD}$ dissertation, Georgia Institute of Technology, Atlanta, 2008.

[13] Southern Standard Building Code, Southern Building Code Congress, Birmingham, AL, 1969.

[14] Southern Building Code Congress International, Standard Building Code, Birmingham, AL, 1991.

[15] Elnashai, A.S., Papanikolau V. \& Lee D.H., ZEUS Non-linear: A System for Inelastic Analysis of Structures V1.9.0, UIUC, Urbana-Champaign, IL, 2011.

[16] Rix, G.J. \& Fernandez-Leon, J.A., Synthetic ground motions for Memphis, TN, MidAmerica Earthquake Center, 2008.

[17] Mazzoni, S., McKenna, F., Scott, M.H., Fenves, G.L., et al. Open system for earthquake engineering simulation (OpenSees). Pacific Earthquake Engineering Research Center. Version $1.7 .3 ; 2008$.

[18] Nielson, B.G., Analytical fragility curves for highway bridges in moderate seismic zones. Ph.D. dissertation, School of Civil and Environmental Engineering, Georgia Institute of Technology, Atlanta, 2005. 